\title{
3D BUILDING RECONSTRUCTION FROM LIDAR POINT CLOUDS BY ADAPTIVE DUAL CONTOURING
}

\author{
E. Orthuber ${ }^{\mathrm{a}, \mathrm{b}}$, J. Avbelj ${ }^{\mathrm{a} *}$ \\ ${ }^{\text {a }}$ German Aerospace Center, Oberpfaffenhofen, D-82234 Wessling - janja.avbelj@ dlr.de \\ ${ }^{\mathrm{b}}$ Dept. of Remote Sensing Technology, Technische Universitaet Muenchen, Arcisstr. 21, D-80333 Muenchen
}

KEY WORDS: LIDAR, Building, City, Model, Reconstruction, Computer, Vision, Photogrammetry

\begin{abstract}
:
This paper presents a novel workflow for data-driven building reconstruction from Light Detection and Ranging (LiDAR) point clouds. The method comprises building extraction, a detailed roof segmentation using region growing with adaptive thresholds, segment boundary creation, and a structural 3D building reconstruction approach using adaptive 2.5D Dual Contouring. First, a 2D-grid is overlain on the segmented point cloud. Second, in each grid cell 3D vertices of the building model are estimated from the corresponding LiDAR points. Then, the number of 3D vertices is reduced in a quad-tree collapsing procedure, and the remaining vertices are connected according to their adjacency in the grid. Roof segments are represented by a Triangular Irregular Network (TIN) and are connected to each other by common vertices or - at height discrepancies - by vertical walls. Resulting 3D building models show a very high accuracy and level of detail, including roof superstructures such as dormers. The workflow is tested and evaluated for two data sets, using the evaluation method and test data of the "ISPRS Test Project on Urban Classification and 3D Building Reconstruction" (Rottensteiner et al., 2012). Results show that the proposed method is comparable with the state of the art approaches, and outperforms them regarding undersegmentation and completeness of the scene reconstruction.
\end{abstract}

\section{INTRODUCTION}

\subsection{Motivation}

For more than two decades, 3D building reconstruction has been an active research topic of remote sensing, photogrammetry, and computer vision (Rottensteiner et al., 2012; Wang, 2013; Haala and Kada, 2010; Lafarge and Mallet, 2012). Continuing research is driven by the increasing demand for accurate, automatically produced, and detailed 3D city models (Wang, 2013). City models are used for urban planning (Verma et al., 2006), change detection (Rau and Lin, 2011) and environmental or telecommunication simulations (Geibel and Stilla, 2000; Rau and Lin, 2011). Today's utilization of city models expands to everyday user-driven mobile applications, such as location based services (Wang, 2013; Brenner, 2005), 3D Geographic Information Systems (GIS) for navigation, driver assistance systems, virtual tourism (Zhou and Neumann, 2010), and augmented reality. The effort for keeping $3 \mathrm{D}$ city models up-to-date depends on the level of automation in building reconstruction.

LiDAR point clouds are well suited for automatic building reconstruction. In comparison to optical stereo imagery, where stereo matching is needed to obtain $3 \mathrm{D}$ geometry, LiDAR data contains directly measured, and thus very accurate 3D information (Meng et al., 2010; Haala and Kada, 2010). With continuously increasing LiDAR sensor capacities and point densities, research on building reconstruction has set a focus on LiDAR point clouds (Geibel and Stilla, 2000; Haala and Kada, 2010).

\subsection{Related Work}

Building reconstruction requires the extraction of individual buildings' points from a LiDAR scene. Once buidlings are extracted, there are two main approaches to reconstruction, i.e. model- and data-driven approaches.

\footnotetext{
${ }^{*}$ Corresponding author
}

Model-driven approaches select for each building point cloud, or parts of it the best fitting parametric model and its corresponding parameters from a predefined catalogue (Maas and Vosselman, 1999; Vosselman and Dijkman, 2001; Kada and McKinley, 2009; Haala and Kada, 2010; Zhang et al., 2012). Model-driven reconstruction is robust, effective and fast, because regularization constraints, such as parallelity and orthogonality, are already inherent in the parametric models. However model-driven approaches are limited to the beforehand defined model portfolio and are therefore not flexible to model all roof shapes.

Data-driven approaches connect individual roof segments, which are constructed according to a preliminary segmentation of the building point cloud. Even though data-driven approaches require a high effort for subsequent regularization, they are widely used (e.g. Rottensteiner et al., 2012; Wang, 2013). The advantages of these approaches are a high fit to the input data and flexibility in modeling complex roof shapes. Roof segmentation can be achieved by surface-fitting techniques such as RANSAC (Sohn et al., 2008; Tarsha-Kurdi et al., 2008; Brenner, 2000) or Hough transform (Vosselman and Dijkman, 2001; Sohn et al., 2012; Vosselman et al., 2004), or using region growing methods (Rottensteiner, 2003; Oude Elberink and Vosselman, 2009; Perera et al., 2012; Verma et al., 2006; Nurunnabi et al., 2012; Dorninger and Pfeifer, 2008; Lafarge and Mallet, 2012). Typically, each segment is delimited by a polygonal segment boundary, which is created by using e.g. Alpha-shapes (Dorninger and Pfeifer, 2008; Kada and Wichmann, 2012; Sampath and Shan, 2007; Wang and Shan, 2009), the Voronoi neighborhood (Maas and Vosselman, 1999; Matei et al., 2008; Rottensteiner, 2003) or using a 2D-grid-cell projection (Sun and Salvaggio, 2013; Zhou and Neumann, 2008). Polyhedral 3D models are commonly constructed on the basis of heuristics for extracting and connecting 3D lines along the segment boundaries (Dorninger and Pfeifer, 2008; Vosselman and Dijkman, 2001; Sohn et al., 2008; Rau and Lin, 2011; Rottensteiner, 2003). Structural modeling procedures estimate the coordinates of the building model's 3D vertices by error propagation techniques (Lafarge and Mallet, 2012) or lo- 
cal error minimization (Fiocco et al., 2005) are less frequently used. The latter technique is also used by Zhou and Neumann (2010), who apply a $2.5 \mathrm{D}$ dual contouring algorithm on a point cloud which is segmented into different height layers. The asset of their method is the outstanding flexibility to model complex roof shapes, including non-planar roof segments. However, the algorithm cannot create step edges between roof segments connecting within one roof height layer, which results in a deficiency for modeling superstructures.

\section{METHOD}

The proposed workflow (Fig. 1) adapts the method of Zhou and Neumann (2010) for modeling superstructures. The algorithm is modified for a situation-adaptive estimation of the 3D building model's vertices from a detailed roof segmentation.

Input to the procedure are LiDAR data, clustered into sets of LiDAR points representing different buildings, hereafter referred to as building point clouds. First, the roof points are segmented on the basis of Triangulated Irregular Network (TIN) of the data. Second, a boundary polygon is created for each segmented cluster. Third, vertices of the 3D building model are estimated and connected using an adaptive 2.5D Dual Contouring procedure. Regularization for enhancing model simplicity is not included in this is work.

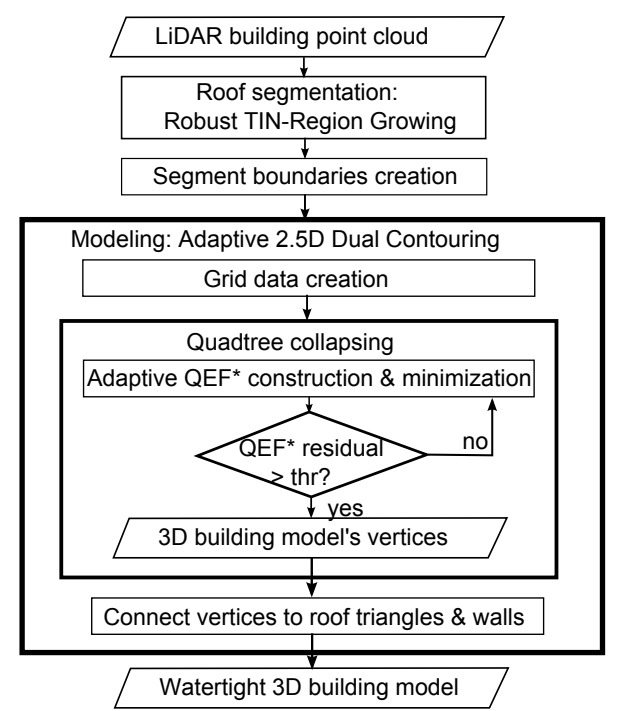

Figure 1. Overview of the proposed workflow for 3D building model reconstruction from LiDAR point clouds. QEF abbreviation stands for Quadratic Error Function (Eq. (4)).

\subsection{Roof Segmentation}

The goal of roof segmentation is to cluster the points according to the roof segments they belong to. While most region growing (RG) segmentation techniques assume roof segments to be planar, the proposed segmentation is designed to allow any continuous shape. For this purpose, a robust TIN-based RG technique is proposed. Moreover, in contrast to most RG techniques (e.g. Dorninger and Pfeifer, 2008; Oude Elberink and Vosselman, 2009; Sampath and Shan, 2010; Sun and Salvaggio, 2013), the proposed method does not assign one segment label to each LiDAR point, but to each triangle of the TIN. Thereby, LiDAR points, which are part of differently labelled triangles, have more than one label. The labelling of triangles minimizes gaps between adjacent intersecting roof segments and allows accurate boundary determination.

The RG procedure iteratively starts at seed triangles defined by a minimum Local Unevenness Factor $(L U F)$, defined as

$$
L U F_{t}=\sum_{k}^{K_{t}}\left(\frac{A_{k}}{\sum_{k}^{K_{t}} A_{k}} \cdot \operatorname{mean}\left(\begin{array}{l}
n_{x, k}-\bar{n}_{x} \\
n_{y, k}-\bar{n}_{y} \\
n_{z, k}-\bar{n}_{z}
\end{array}\right)\right)
$$

where $A_{k}$ is equal to the area and $\mathbf{n}_{k}=\left[n_{x, k}, n_{y, k}, n_{z, k}\right]^{\mathrm{T}}$ is the normal of the $k$-th of $K_{t}$ triangles which are in the neighborhood of triangle $t ; \bar{n}_{x / y / z}$ are the means of all $n_{x / y / z, k}$. For $\mathrm{RG}$, each triangle is tested for a fixed threshold on the local angular deviation and for two adaptive thresholds. These two robust adaptive thresholds are built according to Nurunnabi et al. (2012) and are using the $L U F$ and the LiDAR points' distances to the current best fitting segment plane.

\subsection{Segment Boundaries}

For each point cloud representing a roof segment, a polygonal boundary is created in an iterative convex-hull collapsing procedure. Iteratively, each line segments of the convex hull is refined by the LiDAR point with a minimum distance measure. The refinement stops when the line segment is shorter than a directionally dependent threshold, which is created by considering the LiDAR point spacings in across-track and along-track sampling directions.

\subsection{Building Modeling}

The proposed modeling algorithm estimates and connects the 3D vertices of the building model using an adaptive 2.5D Dual Contouring procedure. In section 2.3.1, the $2.5 \mathrm{D}$ dual contouring principle is introduced. A 2D grid is overlain on the data, and grid data is computed (section 2.3.2). In an iterative quadtree collapsing procedure, a Quadratic Error Function (QEF) is constructed from the grid data of each four adjacent grid cells. The minimization of the situation-adaptive QEF results in the coordinates of one or more 3D vertices of the building model, depending on whether the vertices represent a step- or an intersection edge (section 2.3.3).

2.3.1 Dual Contouring principle For building reconstruction, a $2 \mathrm{D}$ grid is overlain to the segmented LiDAR points in the $\mathrm{x}-\mathrm{y}-$ plane. The Dual Contouring principle can be illustrated by the example of estimating the vertices of boundary polygons, which separate the segments in the 2D-plane. In each grid cell, a polygon vertex is estimated by minimizing its distances from local boundary lines, which separate the LiDAR points of different segments. Then, a polygon is created by connecting the vertices of adjacent cells (Fig. 2).

The purpose of 2.5D Dual Contouring is to estimate vertices of the $3 \mathrm{D}$ building polygon in each grid cell, which are described by so-called hyperpoints. Depending on whether a hyperpoint $\mathbf{X}=\left[\begin{array}{llllll}x & y & z_{v} & z_{v+1} & \ldots & z_{V}\end{array}\right]^{\mathrm{T}}$ describes a step edge or an intersection edge, it contains two or more $3 \mathrm{D}$ vertices with the same $\mathrm{x}$-y-coordinates, but with different z-coordinates. Each zcoordinate defines a $3 \mathrm{D}$ vertex $\mathbf{X}_{3 D, v}=\left[x, y, z_{v}\right]$, in which all segments $S_{k}$ within a local height layer $\mathbf{H}_{v}$ intersect (Fig. 3).

A hyperpoint's optimal $x$ and $y$-coordinates minimize the 2Ddistances $E_{2 D}(\mathbf{X})$ to local boundary lines $(\mathbf{L B L})$ between the respective segments in the $2 \mathrm{D}$ plane. $E_{2 D}(\mathbf{X})$ is computed as 


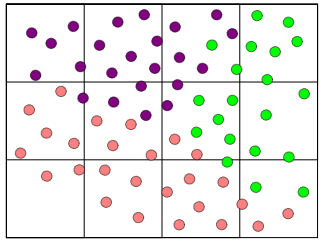

(a) 2D-grid overlain to segmented point cloud. Each colour represents one segment.

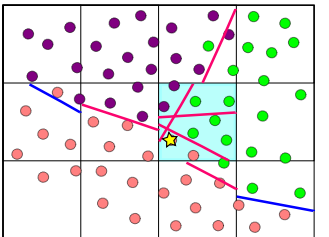

(c) QEF minimization (blue cell) (d) QEF solutions (yellow stars) using all local boundary lines from are computed for all cells and conadjacent cells (pink lines). The nected to the polygonal boundary yellow star is the QEF solution, i.e. line (black line) according to their one optimal vertex of the polygo- adjacency in the grid. nal boundary line.

Figure 2. 2D Illustration of the Dual Contouring principle. The input is segmented point cloud (a) and the QEF solutions are the polygonal boundary lines (d).

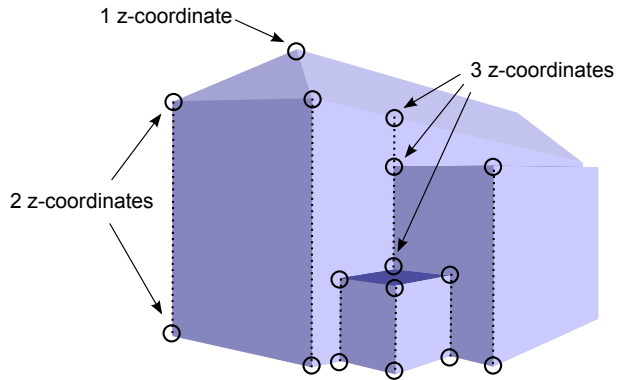

Figure 3. Hyperpoints at intersection edges (one z-coordinate) and step edges (more than one z-coordinate).

$$
E_{2 D}(\mathbf{X})=\sum_{k=1}^{M-1} \sum_{l=2, l \neq k}^{M} \sum_{i_{(k, l)}}^{I_{(k, l)}}\left[\mathbf{n}_{i_{(k, l)}^{\mathrm{T}}}\left(\mathbf{p}_{i_{(k, l)}}-\left[\begin{array}{l}
x \\
y
\end{array}\right]\right)\right]^{2},
$$

where $\mathbf{n}_{i_{(k, l)}}$ is the normal of the $i$-th local boundary line $\mathbf{L B L} \mathbf{L}_{k, l}$ between the segments $S_{k}$ and $S_{l}$, and $\mathbf{p}_{i_{(k, l)}}$ is point on this line.

Additionally, an optimal 3D vertex $\mathbf{X}_{3 D, v}$ corresponding to $\mathbf{H}_{v}$, $v=(1, \ldots, V)$ minimizes the $3 \mathrm{D}$-distances $E_{3 D}(\mathbf{X})$ to the local surface planes (LSP), which are fitted to the LiDAR points belonging to each segment $S_{k}, k=(1, \ldots, M) . E_{3 D}(\mathbf{X})$ is computed as

$$
E_{3 D}(\mathbf{X})=\sum_{H_{v}=1}^{V} \sum_{k \in H_{v}}^{M} \sum_{j_{k}}^{N_{k}}\left[\mathbf{m}_{j_{k}}\left(\mathbf{q}_{j_{k}}-\left[\begin{array}{c}
x \\
y \\
z_{v}
\end{array}\right]\right)\right]^{2},
$$

where $\mathbf{m}_{j_{k}}$ is the $j$-th normal of the local surface plane $\mathbf{L} \mathbf{S P}_{k}$ on segment $S_{k}$, and $\mathbf{q}_{j_{k}}$ is a point on this plane.
Combining $E_{2 D}(\mathbf{X})$ (Eq. 3) and $E_{3 D}(\mathbf{X})$ (Eq. 2), each hyperpoint is estimated by minimizing the Quadratic Error Function (QEF) (Zhou and Neumann, 2010)

$$
\hat{\mathbf{X}}=\underset{\mathbf{X}}{\operatorname{argmin}}\left\{E_{2 D}(\mathbf{X})+E_{3 D}(\mathbf{X})\right\}
$$

2.3.2 Local grid data For each vertex of the $2 \mathrm{D}$ grid, a local surface plane $\mathbf{L S P}=[\mathbf{m}, \mathbf{q}]$ is determined, where $\mathbf{m}=$ $\left[m_{x}, m_{y}, m_{z}\right]^{\mathrm{T}}$ is the plane's normal, and $\mathbf{q}=\left[q_{x}, q_{y}, q_{z}\right]^{\mathrm{T}}$ is a point on the plane. $\mathbf{q}_{x y}=\left[q_{x}, q_{y}\right]^{\mathrm{T}}$ is equal to the grid vertex. Each LSP is associated with a segment label $l_{L S P}$, according to the segment, which is the closest to $\mathbf{q}_{x y}$ (Fig. $4 \mathrm{~b}$ ). Vector $\mathbf{m}$ is determined by averaging the normals of the $K$ nearest TINtriangles belonging to $S_{k}$.

For each grid cell, a local boundary line $\mathbf{L B L}_{k, l}=[\mathbf{n}, \mathbf{p}]$ is estimated for each pair of segments $S_{k}$ and $S_{l}$ using a Least Squares approach (Fig. 4 a). $\mathbf{L B L}_{k, l}$ is estimated from all LiDAR points belonging to $S_{k}$ and $S_{l}$, which are within a buffer zone around the grid cell. $\mathbf{L B L}$ which have no intersection point $\mathbf{p}$ with the grid cell's border are discarded.

\subsubsection{Adaptive QEF In contrast to}

Zhou and Neumann (2010), whose building point cloud is segmented into different height layers, the presented method works on a detailed segmentation of the roof into diffent segments. While Zhou and Neumann (2010) estimate one z-coordinate for each global roof height layer, (eq. 4), the proposed method requires that the LiDAR points within one cell are grouped into local height layers. The advantage of local height layers is that step edges can be created between segments from one global roof height layer. This allows to model complex roof structures such as dormers and shed roof segments. Grouping the segments into local height layers $\mathbf{H}_{v}$ is achieved by estimating a step edge probability SEP for each local boundary line LBL (Eq. 5). Within one cell, all segments with an SEP $<0.5$ are grouped into one local height layer $\mathbf{H}_{v}$. Assuming the z-coordinates of LiDAR points to be normally distributed around their true value, the equation for computing SEP is designed to use the minimum step edge height $T_{\text {step }}$ as standard deviation:

$$
S E P=\exp \left\{-\frac{d_{z}^{2}}{2 \cdot\left(T_{\text {step }}\right)^{2}}\right\},
$$

where $T_{\text {step }}$ is a fixed step edge threshold and $d_{z}$ a measure expressing the local height difference of the two segments.

In Zhou and Neumann (2010), all local boundary lines LBL represent step edges, as their input point cloud is only segmented into global roof height layers. When constructing the QEF, it has to be considered that in the proposed method, LBL can also represent intersection edges. In case of a step edge, ideally only the local boundary lines LBL are considered for estimating a hyperpoint's horizontal position $[x, y]$. The distances to local surface planes LSP should not be considered. As LSP cannot be omitted, a balancing weight $w_{k, l}$ is computed for each group of $\mathbf{n}_{i_{k, l}}, i=1, \ldots, I_{k, l}$ using the corresponding $S E P_{(k, l)}$. Each $w_{k, l}$ ranges from $\left[0, \ldots, 1, \ldots, w_{\max }\right]$, corresponding to a $\operatorname{SEP}(k, l)$ of $[0, \ldots, 0.5, \ldots, 1]$, where $w_{\max }$ is the maximum weight.

If the number of LSP for each roof segment represented in a QEF is not equally distributed, QEF minimization will result in a distortion. For instance, minimizing the $E_{3 D}(\mathbf{X})$ in a QEF containing three $\mathbf{L S P} \mathbf{P}_{k}$ and one $\mathbf{L S P} \mathbf{P}_{k+1}$ will not minimize the point's distances to both roof segments $S_{k}$ and $S_{k+1}$. In order to weight each roof segment equally, each normal $\mathbf{m}_{j_{k}}$ is scaled by the number $N_{k}$ of corresponding $\mathbf{L} \mathbf{S} \mathbf{P}_{\mathbf{k}}$. 


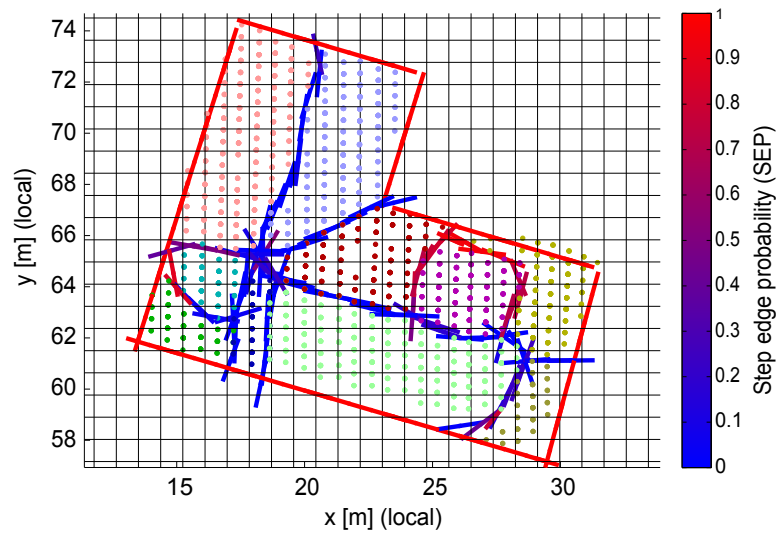

2.3.4 QEF solution and quadtree collapsing Each QEF is solved by least squares adjustment after composing a matrix equation

$$
\hat{\mathbf{X}}_{w}=\underset{\mathbf{X}}{\operatorname{argmin}}\{\mathbf{W}(\mathbf{A X}-\mathbf{b})\}
$$

where $\mathbf{A}$ is the model matix, $\mathbf{b}$ is the vector of observations and $\mathbf{W}$ is the vector containing the weightings of each QEF line, $w_{(k, l)}, k, l=1, \ldots, M, k \neq l$ and $\frac{1}{N_{k}}, k=1, \ldots, M$. Additional solution constraints ensure that the QEF solution lies inside the quadtree cell.

The number of hyperpoints for a building model should me minimized. Therefore, the grid cells are treated as leaf cells of a quadtree, which is iteratively collapsed. For this purpose, the grid is designed to have $2^{n}$ cells. By this, an iterative collapsing of groups of 4 adjacent cells into one larger quadtree cell is possible. For deciding whether to collaps a group of four quadtree (a) Local boundary lines plotted over the segmented LiDAR points. Each colorcells, a combined QEF is constructed from the LSP and LPL of represents one segment. The lines' colors indicate diffferent step edge proba-these cells. If the non weighted residual error $\mathbf{R}_{\mathbf{Q E F}}=\mathbf{A X}-\mathbf{b}$ bilities, ranging from zero (blue) to one (red).

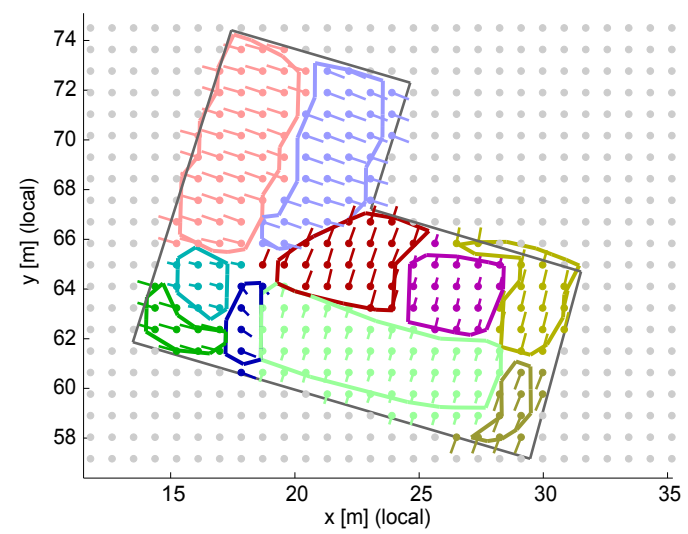

(b) Points $\mathbf{q}$ and normal vectors $\mathbf{m}$ of the local surface planes, plotted over the segment boundaries. Colours correspond to the segment labels $l_{\text {SP. }}$.

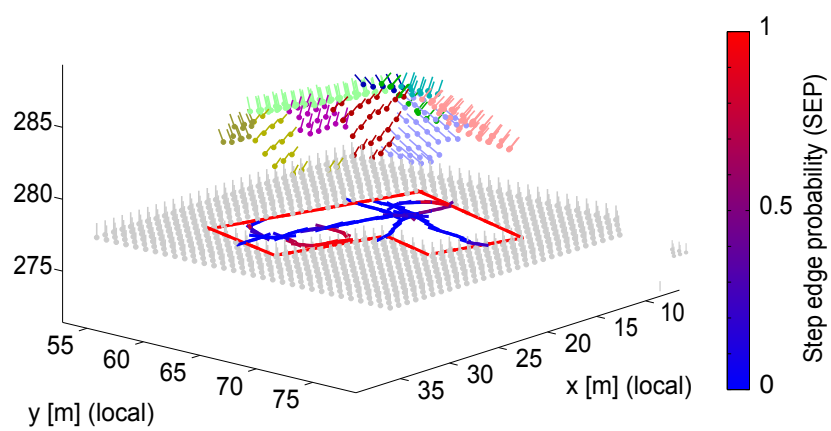

(c) A side view of combined grid data, i.e. local boundary lines LBL and local surface planes LSP.

Figure 4. Local boundary lines and local surface planes in a grid.

The weighted and scaled QEF is defined as

$$
\begin{aligned}
& \hat{\mathbf{X}}_{w}=\underset{\mathbf{X}}{\operatorname{argmin}}\{ \\
& \sum_{k=1}^{M-1} \sum_{l=2, l \neq k}^{M} \sum_{i_{(k, l)}}^{I_{(k, l)}}\left[w_{(k, l)} \cdot \mathbf{n}_{i_{(k, l)}}\left(\mathbf{p}_{i_{(k, l)}}-\left[\begin{array}{l}
x \\
y
\end{array}\right]\right)\right]^{2}+
\end{aligned}
$$

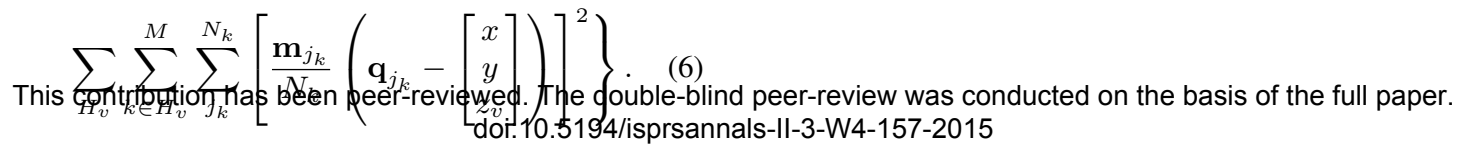

In this section, tests of the proposed method, data and results are

Figure 5. Quads (yellow areas) and triangles (red areas) resulting from connecting adjacent hyperpoint vertices (dark blue points) to 3D edges (blue lines, top view)

Where a 3D edge is only part of one 3D triangle (single edge), a vertical wall has to be created between this $3 \mathrm{D}$ edge and another single edge from the same pair of hyperpoints. If no such other single edge is found, a vertical wall is created to ground. The result is a watertight $3 \mathrm{D}$ building model, represented by a large number of roof triangles and vertical wall elements. Subsequent regularization procedures are recommended for increasing model simplicity, but are not in the scope of this paper.

\section{TESTS AND EVALUATION}$$
\text { The weighted and scaled QEF is defined as }
$$ 


\subsection{Test data and parameters}

Two different datasets are used for testing, a smaller scene from Munich, Germany, and a larger scene from Vaihingen, Germany. The Vaihingen scene corresponds to the test scene "Vaihingen Area 1" of the ISPRS benchmark project. Table 1 shows the data characteristics of the two test scenes and the applied reconstruction parameters.

\begin{tabular}{|c|c|c|}
\hline Test scene & Vaihingen & Munich \\
\hline & \multicolumn{2}{|c|}{ Scene description } \\
\hline pont density [point $/ \mathrm{m}^{2}$ ] & 3.5 & 2.3 \\
\hline vertical accuracy & n.a. & n.a. \\
\hline number of buildings & 21 & 8 \\
\hline number of segments & 182 & 21 \\
\hline & \multicolumn{2}{|c|}{ Reconstruction parameters } \\
\hline step edge theshold $T_{\text {step }}[\mathrm{m}]$ & 0.2 & 0.3 \\
\hline grid cell size $C$ [points / cell] & 2.5 & 3 \\
\hline residual threshold $R[\mathrm{~m}]$ & 0.8 & 1.2 \\
\hline
\end{tabular}

Table 1. Characteristics of the two test scenes and applied parameters for testing the proposed method.

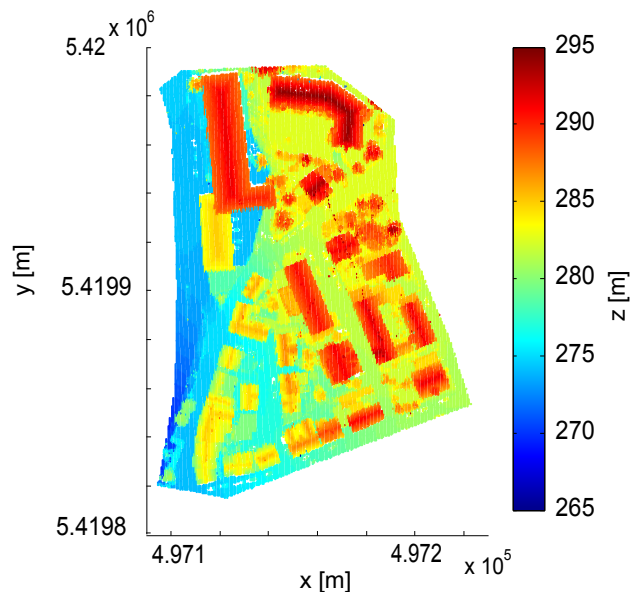

(a) LiDAR point cloud of the Vaihingen test scene.

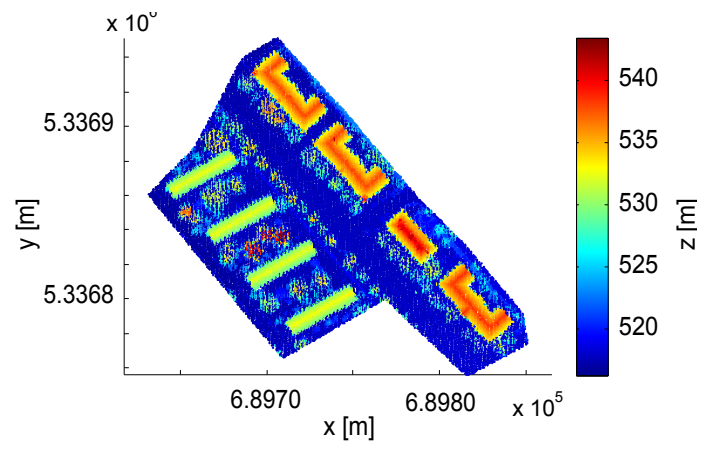

(b) LiDAR point cloud of the Munich test scene.

Figure 6. LiDAR point clouds of the test scenes.

\subsection{Test results}

All the buildings in both datasets were reconstructed. Figure 7 a presents the reconsted city scene of Vaihingen, consisting of 21 buildings. Figure $7 \mathrm{~b}$ presents the reconstructed Munich scene with 8 buildings.

\subsection{Evaluation}

The results of the reconstructed buildings are evaluated according to the evaluation of the the ISPRS benchmark project (Rotten-

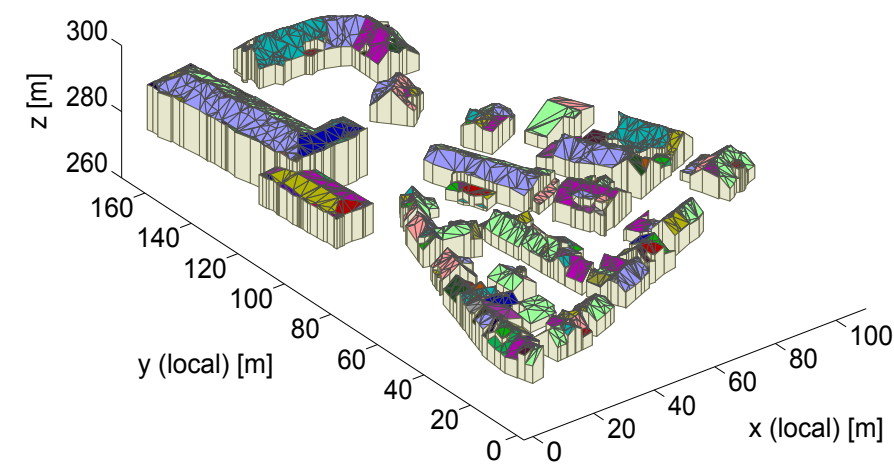

(a) Side view of the reconstructed Vaihingen scene, wall segments (grey areas) are connecting the outer roof boundaries to ground or to other roof segments.

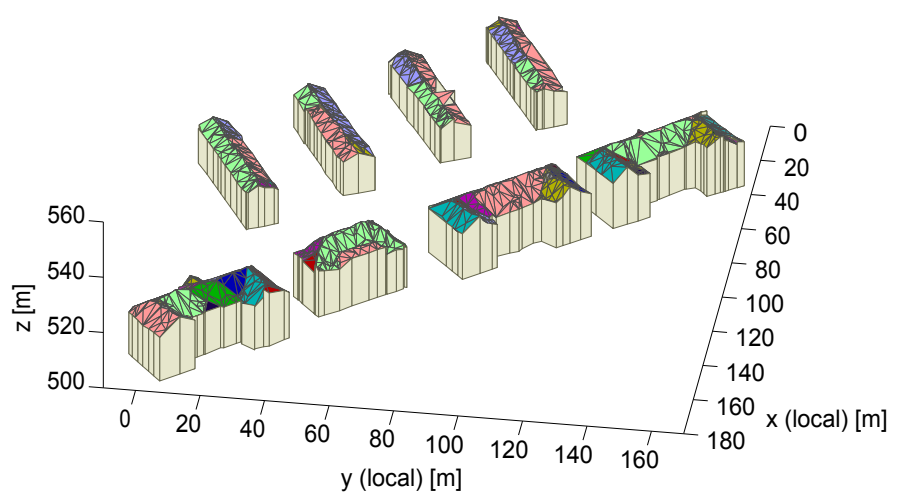

(b) Side view of the reconstructed Munich scene, wall segments (grey areas) are connecting the outer roof boundaries to ground or to other roof segments.

Figure 7. Reconstructed city scenes.

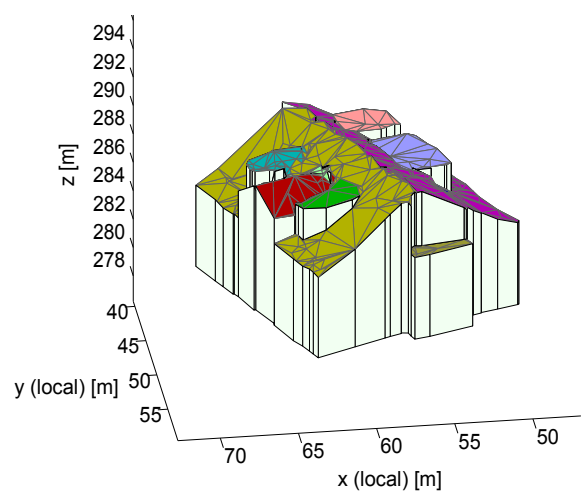

Figure 8. Detailed building model in a local coordinate system. $T_{\text {step }}=0.2 \mathrm{~m}$, grid cell size $C=2.5 \mathrm{LiDAR}$ points per cell, residual threshold $R=0.8 \mathrm{~m}$. Segments from one height layer are separated by both, step edges and intersection edges.

steiner et al., 2012). The ground truth used by the ISPRS benchmark project was not made available. Therefore, the results are evaluated against manually extracted $2 \mathrm{D}$ reference segment polygons. For the Vaihingen data set, ground truth was extracted from the ortho image delivered with the test data for the ISPRS benchmark project. For the Munich data set, ground truth was extracted from high resolution ortho image. The following eight evaluation parameters are calculated:

- Completeness $\mathbf{C}_{\mathbf{m}}=\frac{T P_{r}}{T P_{r}+F N}$, where $T P_{r}$ are true positive and $F N$ are false negative reference polygons, whose area is $\geq 2.5 \mathrm{~m}^{2}$ and which are overlapping by at least $\left(T P_{r}\right.$ 
) or less than $(F N) 50 \%$ with estimated segment polygons. $\mathbf{C}_{\mathbf{m}, 10}$ is computed analogously for segment areas $\geq 10 \mathrm{~m}^{2}$.

- Correctness $\mathbf{C}_{\mathbf{r}}=\frac{T P_{e}}{T P_{e}+F P}$, where $T P_{d}$ are true positive and $F P$ are false positive estimated segments, whose area is $\geq 2.5 \mathrm{~m}^{2}$ and which are overlapping by at least $\left(T P_{d}\right)$ or less than $(F P) 50 \%$ with reference polygons. $\mathbf{C}_{\mathbf{r}, 10}$ is computed analogously for segment areas $\geq 10 \mathrm{~m}^{2}$.

- RMSE computed from the 2D distances $d_{x y}$ of the estimated segment outline vertices to their reference segment, while $d_{x y}>3 \mathrm{~m}$ are neglected.

- No: Number of oversegmented reference segments, i.e. those corresponding to more than one $T P_{d}$.

- $\mathbf{N}_{\mathbf{U}}$ : Number of undersegmenting estimated segsments, i.e. those corresponding to more than one $T P_{r}$.

- $\mathbf{N}_{\mathbf{C}}$ : Number of references which are both under- and oversegmented.

\subsection{Evaluation results}

Tables 2 and 3 show the evaluation parameters for segmentation. 2D segment outlines are evaluated against the reference segments. Results for the Vaihingen scene are compared to those of Awrangjeb and Fraser (2014), who apply the same evaluation method (section 3.3) to their segmentation results.

\begin{tabular}{|l|l|l|}
\hline Method & $\mathbf{C}_{\mathbf{m}}\left(\mathbf{C}_{\mathbf{m}, \mathbf{1 0}}\right)$ & $\mathbf{C}_{\mathbf{r}}\left(\mathbf{C}_{\mathbf{r}, \mathbf{1 0}}\right)$ \\
\hline \multirow{2}{*}{ proposed } & \multicolumn{2}{|c|}{ Munich scene } \\
& $80.4(87.8)$ & $98.6(100.0)$ \\
proposed & \multicolumn{2}{|c|}{ Vaihingen scene } \\
Awrangjeb \& Fraser, 2014 & $91.2(95.0)$ & $93.3(97.3)$ \\
\hline
\end{tabular}

Table 2. Completeness and correctness of the segmentation. Segmentation results of the Vaihingen Scene are compared to the results of Awrangjeb and Fraser (2014).

\begin{tabular}{|l|c|c|c|c|}
\hline Method & $\mathbf{N}_{\mathbf{U}}$ & $\mathbf{N}_{\mathbf{O}}$ & $\mathbf{N}_{\mathbf{C}}$ & RMSE \\
\hline \multirow{3}{*}{ proposed } & \multicolumn{4}{|c|}{ Munich scene } \\
& 3 & 15 & 1 & 1.46 \\
proposed & \multicolumn{4}{|c|}{ Vaihingen scene } \\
Awrangjeb \& Fraser, 2014 & 42 & 25 & 0 & 0.56 \\
\hline
\end{tabular}

Table 3. Under- and oversegmentation and accuracy of the segmentation. Segmentation results of the Vaihingen Scene are compared to the results of Awrangjeb and Fraser (2014).

Tables 4 and 5 show the evaluation results for reconstruction. The outer boundaries of each group of adjacent roof triangles with equal segment label are projected to the $x-y$-plane and evaluated against the reference segments. Results for the Vaihingen scene are compared to those of the ISPRS benchmark project.

It has to be considered that the manually extracted ground truth might differ from the ground truth used by the ISPRS benchmark project.

\section{DISCUSSION}

The proposed method creates building models of high detail by triangulation. In contrast to triangulating the input LiDAR point cloud, adaptive 2.5D Dual Contouring has two main advantages:

\begin{tabular}{|c|c|c|}
\hline Method & $\mathrm{C}_{\mathrm{m}}\left(\mathrm{C}_{\mathrm{m}, \mathbf{1 0}}\right)$ & $\mathbf{C}_{\mathrm{r}}\left(\mathrm{C}_{\mathrm{r}, 10}\right)$ \\
\hline & \multicolumn{2}{|c|}{ Munich scene } \\
\hline \multirow{2}{*}{ proposed } & $96.1(100.0)$ & $98.7(100)$ \\
\hline & \multicolumn{2}{|c|}{ Vaihingen scene } \\
\hline proposed & $100.0(100.0)$ & $95.4(95.4)$ \\
\hline Rau \& Lin, 2011 & $86.7(86.7)$ & $98.9(99.3)$ \\
\hline Elberink \& Vosselman, 2009 & $60.8(58.5)$ & $94.6(94.0)$ \\
\hline Elberink \& Vosselman, 2011 & $65.3(63.3)$ & $97.3(97.3)$ \\
\hline Xiong* & $76.0(72.9)$ & $94.5(95.1)$ \\
\hline Dorninger \& Pfeifer, 2008 & $72.2(77.7)$ & $96.7(96.5)$ \\
\hline Sohn et al., 2008 & $88.2(89.9)$ & $98.5(98.2)$ \\
\hline
\end{tabular}

Table 4. Completeness and correctness of the reconstruction. Results of the Vaihingen Scene are compared to the results of other methods (* see Rottensteiner et al., 2012).

\begin{tabular}{|l|c|c|c|c|}
\hline Method & $\mathbf{N}_{\mathbf{U}}$ & $\mathbf{N}_{\mathbf{O}}$ & $\mathbf{N}_{\mathbf{C}}$ & RMSE \\
\hline & \multicolumn{4}{|c|}{ Munich scene } \\
proposed & 4 & \multicolumn{4}{|c|}{ Vaihingen scene } \\
proposed & 13 & 32 & 1 & 0.72 \\
Rau \& Lin, 2011 & 36 & 10 & 3 & 0.66 \\
Elberink \& Vosselman, 2009 & 26 & 16 & 17 & 0.91 \\
Elberink \& Vosselman, 2011 & 38 & 0 & 3 & 0.94 \\
Xiong* & 40 & 2 & 2 & 0.84 \\
Dorninger \& Pfeifer, 2008 & 42 & 7 & 6 & 0.79 \\
Sohn et al., 2008 & 36 & 5 & 14 & 0.75 \\
\hline
\end{tabular}

Table 5. Under- and over-segmentation and accuracy of the reconstruction. Results of the Vaihingen Scene are compared to the results of other methods (* see Rottensteiner et al., 2012).

Depending on the chosen level of detail (by setting the parameters $C$ and $R$ ), points for building representation are reduced to a minimum at continuities. Second, step edges are always represented as vertical walls, as vertices of a hyperpoint are always vertically arranged.

The novelty of the proposed reconstruction method is using a detailed segmentation as input to a 2.5D Dual Contouring approach. In contrast to Zhou and Neumann (2010), the proposed situationadaptive QEF construction allows to model accurate step edges between segments from one height layer (Fig. 8). For each pair of local height layers within each grid cell, a decision is made whether to connect them either by an intersection edge or by a step edge. The step edge threshold $T_{\text {step }}$, the residual for quadtree collapsing $R$, and the grid cell size $C$ are of equal importance for deciding whether a step edge or an intersection edge is locally created. If $C$ is chosen too high, roof segments which require both intersection edges and step edges to be modelled accurately (e.g. dormers) will only fall into one height layer and are "smoothed out". The same smoothing effect happens if $R$ or $T_{\text {step }}$ are chosen too high, or if roof segments within one roof heigh layer are not segmented accurately, i.e. undersegmentation occurs (Fig. 9).

Zhou and Neumann (2010) do not carry out additional roof height layer segmentation. Consequnetly, no step edges can be created between parts of one height layer. In contrast, the presented adaptive 2.5D Dual Contouring method can model superstructures such as dormers by allowing both intersection and step edges within one height layer.

A further advantage of the proposed method is that the building models' level of detail can be influenced by changing $C$ and $R$. Thereby, a trade-off between the desired level of detail on the one side, and the computation time and the number of hyperpoints in a building model on the other side has to be made. Smaller $C$ and smaller $R$ result in larger number of hyperpoints and thus in 


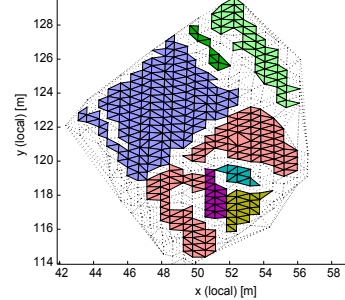

(a) Undersegmentation of the blue segment. Opposite the dark green segment, a similar separate roof part should have been segmented.
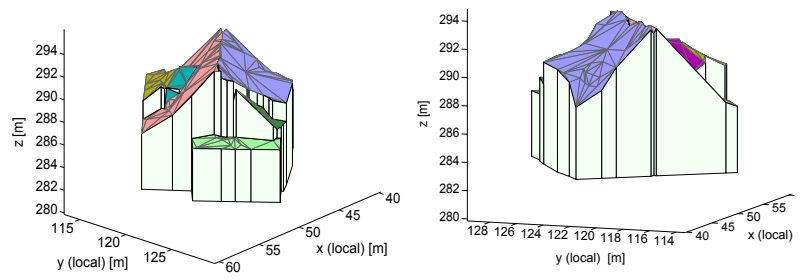

(b) Reconstruction of a step edge (c) Reconstruction, where no step between the blue and the dark green edge can be modelled, because no segment. different segments are identified.

Figure 9. Smoothed roof effect due to undersegmentation. If the roof height layer is undersegmented no step edges can be created, because only one local height layers is identified. However, the roof is approximated to fit the input point cloud in detail.

more roof triangles. However, if a regularized building model is required the large number of roof triangles increases the effort for postprocessing.

The building model's 3D edges are constructed from connections of adjacent hyperpoints in the quadtree. In certain cases, this can lead to erroneous edges (Fig. 10b, right arrow). If hyperpoints from non-adjacent quadtree cells are connected to an edge, deformations or "stand-alone walls" in the model can occur (Fig. $10 \mathrm{~b}$, left arrow).

\section{CONCLUSION}

A novel method for creating detailed building models with complex roof shapes from LiDAR point clouds is proposed in this paper. The 2.5D Dual Contouring method of Zhou and Neumann (2010) is used and adapted in a way that step edges and intersection edges can be created between roof segments. A main contribution of this work is the modification and weighting of the Quadratic Error Function (QEF) for modeling step edges and intersection edges. The modeling depends on the step edge probabilities of local height layers. A prerequisite for adaptive 2.5D Dual Contouring is a roof segmentation technique which stops at smooth edges. The applied robust TIN-based region growing reliably stops at smooth edges. Consequently, undersegmentation is significantly reduced. The resulting building models show a very high fit to the input LiDAR points. Each roof segment is represented by a triangulation, thus also non-planar roof shapes can be modelled. Subsequent model regularization is recommended, because buildings are represented by a large number of vertices. Errors in reconstruction result mostly from wrong or missing connections of the vertices. Thus, the way the connections of the vertices to the building model should be more robust. Wrong connections could be avoided by checking for the consistency of the model with the building footprint. Under assumption that building edges are mostly orthogonal or parallel to the main build-

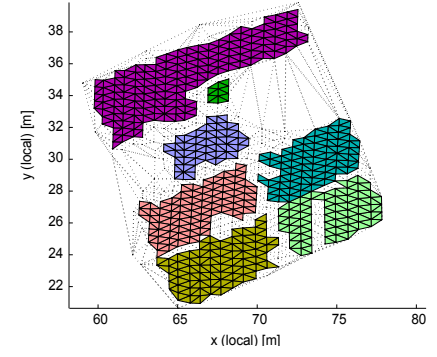

(a) Segmentation of the building point cloud.

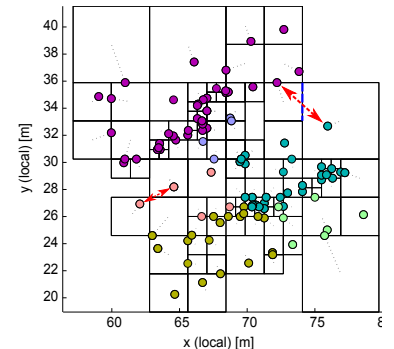

(b) One hyperpoint (dots) computed for each cell of the collapsed quadtree. Erroneously connected hyperpoints of neighboring quadtree cells (right arrow), and of non-neighboring hyperpoints (left arrow).

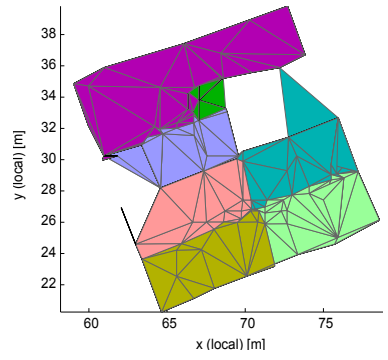

(c) 3D model resulting from erroneous hyperpoint connection. An additional (wrong) rooftop triangle due to wrong hyperpoint connection (right part), and a "standalone wall" due to missing hyperpoint connection (left).
Figure 10. Errors due to connectivity of hyperpoints from (non)neighboring quadtree cells.

ing direction, the missing connections could be avoided by aligning the building point cloud to the main building direction before the modeling procedure. The proposed workflow was tested and evaluated using two data sets with different characteristics and varying building complexity. Evaluation of the results has shown that both segmentation of LiDAR point clouds and reconstruction of buildings are comparable to the state-of-the-art methods.

\section{ACKNOWLEDGEMENTS}

The Vaihingen data set was provided by the German Society for Photogrammetry, Remote Sensing and Geoinformation (DGPF) Cramer (2010):

http://www.ifp.uni-stuttgart.de/dgpf/DKEP-Allg.html.

\section{References}

Awrangjeb, M. and Fraser, C. S., 2014. Automatic segmentation of raw lidar data for extraction of building roofs. Remote Sensing 6(5), pp. 3716-3751.

Brenner, C., 2000. Towards fully automatic generation of city models. International Archives of Photogrammetry, Remote Sensing and Spatial Information Sciences 33(B3/1), pp. 8492.

Brenner, C., 2005. Building reconstruction from images and laser scanning. International Journal of Applied Earth Observation and Geoinformation 6(3), pp. 187-198.

Cramer, M., 2010. The DGPF-test on digital airborne camera evaluationoverview and test design. PhotogrammetrieFernerkundung-Geoinformation 2010(2), pp. 73-82. 
Dorninger, P. and Pfeifer, N., 2008. A comprehensive automated $3 \mathrm{D}$ approach for building extraction, reconstruction, and regularization from airborne laser scanning point clouds. Sensors 8(11), pp. 7323-7343.

Fiocco, M., Bostrom, G., Gonalves, J. G. and Sequeira, V., 2005. Multisensor fusion for volumetric reconstruction of large outdoor areas. In Proc. of the Fifth IEEE International Conference on 3-D Digital Imaging and Modeling 3DIM pp. 47-54.

Geibel, R. and Stilla, U., 2000. Segmentation of laser altimeter data for building reconstruction: different procedures and comparison. International Archives of Photogrammetry, Remote Sensing and Spatial Information Sciences 33(B3/1), pp. 326334

Haala, N. and Kada, M., 2010. An update on automatic 3D building reconstruction. ISPRS Journal of Photogrammetry and Remote Sensing 65(6), pp. 570-580.

Kada, M. and McKinley, L., 2009. 3D building reconstruction from lidar based on a cell decomposition approach. International Archives of Photogrammetry, Remote Sensing and Spatial Information Sciences 38(3), W4, pp. 47-52.

Kada, M. and Wichmann, A., 2012. Sub-surface growing and boundary generalization for 3D building reconstruction. ISPRS Annals of the Photogrammetry, Remote Sensing and Spatial Information Sciences I-3, pp. 233-238.

Lafarge, F. and Mallet, C., 2012. Creating large-scale city models from 3D-point clouds: A robust approach with hybrid representation. International Journal of Computer Vision 99(1), pp. 69-85.

Maas, H. G. and Vosselman, G., 1999. Two algorithms for extracting building models from raw laser altimetry data. ISPRS Journal of Photogrammetry and Remote Sensing 54(2), pp. 153-163.

Matei, B. C., Sawhney, H. S., Samarasekera, S., Kim, J. and Kumar, R., 2008. Building segmentation for densely built urban regions using aerial lidar data. In Proc. of the IEEE Conference on Computer Vision and Pattern Recognition (CVPR) pp. 1-8.

Meng, X., Currit, N. and Zhao, K., 2010. Ground filtering algorithms for airborne lidar data: A review of critical issues. Remote Sensing 2(3), pp. 833-860.

Nurunnabi, A., Belton, D. and West, G., 2012. Robust segmentation in laser scanning 3D point cloud data. In Proc. of the IEEE International Conference on Digital Image Computing Techniques and Applications (DICTA) 1, pp. 1-8.

Oude Elberink, S. and Vosselman, G., 2009. Building reconstruction by target based graph matching on incomplete laser data: analysis and limitations. Sensors 9(8), pp. 6101-6118.

Perera, S. N., Nalani, H. A. and Maas, H. G., 2012. An automated method for 3D roof outline generation and regularization in airbone laser scanner data. ISPRS Annals of the Photogrammetry, Remote Sensing and Spatial Information Sciences I-3, pp. 281-286.

Rau, J. Y. and Lin, B. C., 2011. Automatic roof model reconstruction from als data and $2 \mathrm{~d}$ ground plans based on side projection and the tmr algorithm. ISPRS Journal of Photogrammetry and Remote Sensing 66(6), pp. 13-27.

Rottensteiner, F., 2003. Automatic generation of high-quality building models from lidar data. IEEE Computer Graphics and Applications 23, pp. 42-50.
Rottensteiner, F., Sohn, G., Jung, J., Gerke, M., Baillard, C., Benitez, S. and Breitkopf, U., 2012. The ISPRS benchmark on urban object classification and 3D building reconstruction. ISPRS Annals of Photogrammetry, Remote Sensing and Spatial Information Sciences I-3, pp. 293-298.

Sampath, A. and Shan, J., 2007. Building boundary tracing and regularization from airborne lidar point clouds. Photogrammetric Engineering and Remote Sensing 73(7), pp. 805-812.

Sampath, A. and Shan, J., 2010. Segmentation and reconstruction of polyhedral building roofs from aerial lidar point clouds. IEEE Transactions on Geoscience and Remote Sensing 48(3), pp. 1554-1567.

Sohn, G., Huang, X. and Tao, V., 2008. Using a binary space partitioning tree for reconstructing polyhedral building models from airborne lidar data. Photogrammetric Engineering and Remote Sensing 74(11), pp. 1425-1438.

Sohn, G., Jwa, Y., Jung, J. and Kim, H., 2012. An implicit regularization for 3D building rooftop modeling using airborne lidar data. ISPRS Annals of Photogrammetry, Remote Sensing and Spatial Information Sciences I-3, pp. 305-310.

Sun, S. and Salvaggio, C., 2013. Aerial 3D building detection and modeling from airborne lidar point clouds. IEEE Journal of Selected Topics in Applied Earth Observations and Remote Sensing 6(3), pp. 1440-1449.

Tarsha-Kurdi, F., Landes, T. and Grussenmeyer, P., 2008. Extended ransac algorithm for automatic detection of building roof planes from lidar data. The photogrammetric journal of Finland 21(1), pp. 97-109.

Verma, V., Kumar, R. and Hsu, S., 2006. 3D building detection and modeling from aerial lidar data. In Proc. of the IEEE Computer Society Conference on Computer Vision and Pattern Recognition 2, pp. 2213-2220.

Vosselman, G. and Dijkman, S., 2001. 3D building model reconstruction from point clouds and ground plans. International Archives of Photogrammetry Remote Sensing and Spatial Information Sciences 34(3/W4), pp. 37-44.

Vosselman, G., Gorte, B. G., Sithole, G. and Rabbani, T., 2004. Recognising structure in laser scanner point clouds. International Archives of Photogrammetry Remote Sensing and SpatialInformation Sciences 46(8), pp. 33-38.

Wang, J. and Shan, J., 2009. Segmentation of lidar point clouds for building extraction. In Proc. of the Annual Conference of the American Society for Photogrammetry and Remote Sensing pp. 9-13.

Wang, R., 2013. 3D building modeling using images and lidar: a review. International Journal of Image and Data Fusion 4(4), pp. 273-292.

Zhang, W., Chen, Y., Yan, K., Yan, G. and Zhou, G., 2012. Primitive-based 3D building reconstruction method tested by reference airborne data. International Archives of the Photogrammetry, Remote Sensing and Spatial Information Sciences 39(B3), pp. 373-378.

Zhou, Q. Y. and Neumann, U., 2008. Fast and extensible building modeling from airborne lidar data. In Proc. of the 16th International Conference on Advances in Geographic Information Systems, ACM GIS 2008 (on CD-ROM).

Zhou, Q. Y. and Neumann, U., 2010. 2.5 d dual contouring: a robust approach to creating building models from aerial lidar point clouds. In Proc. of the European Conference on Computer Vision (ECCV) pp. 115-128. 